\title{
X-ray observations of RX J0822-4300 and Puppis-A
}

\author{
C. Y. Hui and W. Becker \\ Max-Planck-Institut für Extraterrestrische Physik, 85741 Garching bei München, Germany \\ Received 2 June 2005 / Accepted 24 March 2006

\begin{abstract}
Based on observations with the X-ray observatories Chandra and XMM-Newton we present results from a detailed spectro-imaging and timing analysis of the central compact X-ray source RX J0822-4300 in the supernova remnant Puppis-A. The superior angular resolution of Chandra allows for the first time to pinpoint the point source nature of this object down to $0.59 \pm 0.01$ arcsec $(F W H M)$ and to determine its position: RA $=08^{\mathrm{h}} 21^{\mathrm{m}} 57.40^{\mathrm{s}}$, Dec $=-43^{\circ} 00^{\prime} 16.69^{\prime \prime}(\mathrm{J} 2000)$ with sub-arcsecond accuracy. Spectral fits based on Chandra and XMM-Newton data provide a tight constraint on the emission properties of RX J0822-4300. Most of its X-ray emission seems to be of thermal origin. A model spectrum consisting of two blackbody components with $T_{1} \simeq 2.6 \times 10^{6} \mathrm{~K}, T_{2} \simeq 5.0 \times 10^{6} \mathrm{~K}$ and $R_{1} \simeq 3.3 \mathrm{~km}, R_{2} \simeq 0.75 \mathrm{~km}$ for the blackbody temperatures and the size of the projected emitting regions, respectively, provides the best model description of its spectrum. A search for X-ray pulsations from RX J0822-4300 revealed an interesting periodicity candidate which, if confirmed, does not support a scenario of steady spin-down.
\end{abstract}

Key words. pulsars: individual: RX J0822-4300 - stars: neutron - supernovae: individual: Puppis-A - X-rays: stars

\section{Introduction}

For many years, it has been generally believed that all young neutron stars have similar properties as those observed in young rotation-powered pulsars, i.e. emitting strongly pulsed plus powerful plerionic radiation caused by non-thermal emission processes in the neutron star's magnetosphere. Many recent observations of compact X-ray sources in supernova remnants (SNRs), however, suggest that this picture is incomplete and no longer justified. Apart from appearing as rotation-powered pulsars, it has been shown that there are other manifestations of young neutron stars, e.g. with no radio counterpart identified. There are slowly rotating $(P \sim 6-12 \mathrm{~s})$ compact objects which possibly have an ultra strong $\left(B \sim 10^{14}-10^{15} \mathrm{G}\right)$ magnetic field. These neutron stars are dubbed as magnetars, which include the anomalous X-ray pulsars (AXPs) and the soft gamma-ray repeaters (SGRs), depending on whether bursts of strong $\gamma$-ray emission is detected from them (e.g., Mereghetti 1998; Thompson 2000). The other class of objects are the "radio-quiet neutron stars" (e.g. Brazier \& Johnston 1999). Most of them were identified by their high X-ray to optical flux ratios, others simply by their locations near to the expansion centers of SNRs (e.g. Becker \& Pavlov 2001; Kaspi et al. 2004), strongly suggesting that they are indeed the compact stellar remnants formed in the supernova events. The group of SNRs which are known to host a radio-quiet but X-ray bright central compact object is a slowly growing one. Thanks to more sensitive X-ray observatories it currently includes Cas-A (Tananbaum 1999), the Vela-Jr. remnant (RX J0852.0-4622; Aschenbach 1998), RX J1713.7-3946 (Pfeffermann \& Aschenbach 1996), RCW 103 (Tuhoy \& Garmire 1980), Puppis-A (Petre et al. 1982), PKS 1209-51/52 (Helfand \& Becker 1984) and Kes 79 (Seward et al. 2003; Gotthelf et al. 2005).

The discovery of the X-ray point source, RX J0822-4300, in Puppis-A was initially made in one of the EINSTEIN HRI images of the SNR G260.4-3.4 (Petre et al. 1982).
RX J0822-4300 appeared in this data as a faint $X$-ray feature. With ROSAT, it became strongly evident that RX J0822-4300 is the compact stellar remnant which was formed in the $\mathrm{SN}$ event (Petre et al. 1996; hereafter PBW96), although the positional offset from the SNR's optical expansion center is 6.1 arcmin (cf. Winkler \& Kirshner 1985; Winkler et al. 1988). The age of Puppis-A, estimated from the kinematics of oxygen-rich filaments is $\sim 3700$ years. The remnant's kinematic distance estimated from an HI study of the interstellar medium along the line of sight towards Puppis-A is $\sim 2.2 \pm 0.3 \mathrm{kpc}$ (Reynoso et al. 1995, 2003). The space velocity of RX J0822-4300 required to travel to its observed position thus is $\sim 1000 \mathrm{~km} \mathrm{~s}^{-1}$. This is very high if compared with the mean proper motion velocity observed in ordinary field pulsars but still comparable with what is observed in several of the other young supernova/pulsar associations (Manchester et al. 2005).

RX J0822-4300 has not been detected as a radio pulsar. Limiting radio flux densities at $436 \mathrm{MHz}, 660 \mathrm{MHz}$ and $1520 \mathrm{MHz}$ are $1.5 \mathrm{mJy}, 1.3 \mathrm{mJy}$ and $0.3 \mathrm{mJy}$, respectively (Kaspi et al. 1996). For comparison, the limiting flux density in the Parkes Multi Beam Survey along the galactic plane was $\approx 0.2 \mathrm{mJy}$ (Manchester et al. 2001) and the typical limiting sensitivity in deep searches for young radio pulsars in SNRs is $50 \mu \mathrm{Jy}$ (Camilo 2003). Gaensler et al. (2000) searched for a radio nebula around RX J0822-4300 with a resolution of $27 \times 17$ arcsec. Their non-detection of any extended plerionic radio emission up to a scale of 30 arcmin prompted them to conclude that if RX J0822-4300 is a rotation-powered pulsar, e.g. with the radio beam not intersecting with the observer's line of sight, then it must be less powerful than other typical young radio pulsars located in SNRs. All young radio pulsars which are associated with a SNR have a spin-down power in excess of $\sim 10^{36} \mathrm{ergs} / \mathrm{s}$ and are observed to power a X-ray/radio bright pulsar-wind nebula.

RX J0822-4300 has no optical counterpart down to a limiting magnitude of $B \gtrsim 25.0$ and $R \gtrsim 23.6$ (PBW96). This limit 
yields an X-ray-to-optical flux ratio $f_{\mathrm{X}} / f_{B} \gtrsim 5000$ (PBW96). Together with the radio upper limits this rules out many types of X-ray sources as a likely counterpart of RX J0822-4300, except a neutron star.

PBW96 fitted the ROSAT PSPC spectrum with a blackbody model and obtained a temperature of $(3.2 \pm 1.2) \times 10^{6} \mathrm{~K}$ and a column density of $(4.1 \pm 0.2) \times 10^{21} \mathrm{~cm}^{-2}$. The radius of the corresponding blackbody emitting area in their fits is only $\sim 2 \mathrm{~km}$. Zavlin et al. (1999; hereafter ZTP99) tested whether a hydrogen atmosphere model could bring this result in better agreement with the predictions of standard cooling models. They fitted a temperature which is about half that found by PBW96 though with an increased radius of $10 \mathrm{~km}$ for the emitting area. However, as atmosphere models are seen not to be in agreement with the spectral fits from the cooling neutron stars Geminga, PSR B0656+14 and PSR B1055-52 (e.g. De Luca et al. 2005), the applicability of those models which in most cases use nonmagnetic opacities only, is restricted.

EINSTEIN and ROSAT data do not show any evidence for short or long term flux variations. Although a marginal detection of X-ray pulses at a period of $\sim 75.3 \mathrm{~ms}$ was claimed by Pavlo et al. (1999; hereafter PZT99), it could not be confirmed so far (Pavlov et al. 2002; Becker \& Aschenbach 2002).

In order to put tighter constraints on the emission properties of RX J0822-4300, various observations with the new generation X-ray satellites XMM-Newton and Chandra were targeted to it in the past few years. Making use of XMM-Newton's huge collecting power and high spectral resolution as well as of Chandra's sub-arcsecond angular resolution we have performed a sensitive broadband spectro-imaging analysis of RX J0822-4300 and its environment using all XMM-Newton and Chandra data taken from this source so far. This is the subject of this paper which is organized as follows. In Sect. 2 we give a brief description of the relevant XMM-Newton and Chandra observations. In Sect. 3, we present the methods and results of our data analysis which are discussed in Sect. 4 in the context of a number of physical models for the nature of RX J0822-4300.

\section{Observations}

In total, five observations have been targeted with XMM-Newton and Chandra on RX J0822-4300. All data have been taken between December 1999 and November 2001. We summarize the basic information of these observations in Table 1 and give a more detailed description in the following subsections.

\subsection{XMM-Newton observations}

Two of the five data sets reported here were obtained with the European Photon Imaging Camera (EPIC) aboard XMM-Newton (Jansen et al. 2001). EPIC consists of two Metal Oxide Semiconductor (MOS1/2) CCD detectors (Turner et al. 2001) of which half of the beam from two of the three $\mathrm{X}$-ray telescopes is reflected to. The other two halves of the incoming photon beams are reflected to a grating spectrometer (RGS) (den Herder et al. 2001). The third of the three X-ray telescopes is dedicated to expose the EPIC-PN CCD detector solely (Strüder et al. 2001). The April 2001 XMM-Newton observation (hereafter XMM1) was taken with a total exposure time of $\sim 28.8 \mathrm{ks}$. The November 2001 observation (hereafter XMM2) had an exposure time of $\sim 24.3 \mathrm{ks}$. The EPIC-PN CCD was operated in both observations in small-window mode with a thin filter to block optical stray light. This data provide imaging, spectral and temporal information. All recorded events are time tagged with a temporal resolution of $5.7 \mathrm{~ms}$. The MOS1/2 CCDs were setup to operate in full-window mode with a medium filter in the April 2001 observation and a thick filter in the November 2001 observation. The MOS1/2 cameras provide imaging, spectral and timing information, though the later with a temporal resolution of $2.6 \mathrm{~s}$ only.

For both XMM-Newton observations, the satellite was pointed to RA $=08^{\mathrm{h}} 21^{\mathrm{m}} 56^{\mathrm{s}}$ and Dec $=-43^{\circ} 00^{\prime} 19^{\prime \prime}$ [J2000]) which places RX J0822-4300 at the optical axis in the EPIC-PN CCDs. The raw data from the EPIC instruments were processed with version 6.0.0 of the XMM Science Analysis Software. Examining the raw data from the EPIC-PN CCD for both XMM1 and XMM2, we did not find any timing anomaly observed in many of the XMM-Newton data sets (cf. Becker \& Aschenbach 2002; Kirsch et al. 2004). This provides us with opportunities for an accurate timing analysis. We created filtered event files for the energy range $0.3 \mathrm{keV}$ to $10 \mathrm{keV}$ for all EPIC instruments. A small fraction of X-ray events might be split between CCD pixels. In order to correct for this effect only those events were accepted for which the corresponding X-ray generated pattern was between 0-12 in MOS cameras and between $0-4$ in the EPIC-PN camera ${ }^{1}$. We further cleaned the data by accepting only the good times when sky background was low and removed all events potentially contaminated by bad pixels. The effective exposure times after data cleaning are summarized in Col. 7 of Table 1.

In order to correct for the non-uniformity across the detector and the mirror vignetting, exposure map is needed to rescale all parts of the image to the same relative exposure. This is created by using XMMSAS task EEXPMAP.

RX J0822-4300 is located in a patchy SNR environment. This makes the extraction of its source and background spectrum difficult. In order to maximize the signal-to-noise ratio for RX J0822-4300, we extracted its source spectrum from circles with 18 arcsec radii in both, the MOS1/2 and EPIC-PN cameras. About $70 \%$ of all point source events are located within the selection region. Annular regions with radii between 20-35 arcsec, centered at RX J0822-4300, were used to extract the background spectra. The background corrected count rates are listed in Col. 8 of Table 1 . Response files were computed for all data sets by using the XMMSAS tasks RMFGEN and ARFGEN.

\subsection{Chandra observations}

Three of the five data sets on RX J0822-4300 were taken with the Chandra satellite (e.g. Weisskopf 2004). One observation was performed by using the Advanced CCD Imaging Spectrometer (ACIS; Burke et al. 1997) whereas the other two exposures were done by using the High Resolution Camera (HRC; Zombeck et al. 1995; Murray et al. 1997). For the data reduction we used CIAO 3.0.2.

The Chandra HRC data were taken on 1999 December 21 and 2001 January 25 for HRC-I and HRC-S, respectively, with RX J0822-4300 placed $\sim 0.3$ arcmin off-axis. In order to determine the event positions accurately, we started the analysis with level-1 event files and corrected for the tap-ringing distortion in the HRC event position reconstruction. Apart from this, we also performed the de-gap correction to the event files so as to compensate the systematic errors introduced in the event positions by

1 For a detailed description of EPIC event grade selection, please see the XMM-Newton Users' Handbook. 
Table 1. Details of the XMM-Newton and Chandra observations of RX J0822-4300.

\begin{tabular}{llllcccc}
\hline \hline Telescope & Instrument & Instrument mode & Filter & Obs.ID & Start date & $\begin{array}{c}\text { Effective } \\
\text { exposure }\end{array}$ & $\begin{array}{l}\text { Net rate } \\
\left(\mathrm{cts} \mathrm{s}^{-1}\right)\end{array}$ \\
\hline Chandra & HRC-I & Default & UVIS & 749 & $1999-12-21$ & $16 \mathrm{ks}$ & $0.21 \pm 0.01$ \\
Chandra & ACIS-S 2 & Timed Exposure & OBF & 750 & $2000-01-01$ & $11 \mathrm{ks}$ & $0.71 \pm 0.01$ \\
Chandra & HRC-S & Imaging & UVIS & 1851 & $2001-01-25$ & $19 \mathrm{ks}$ & $0.32 \pm 0.01$ \\
XMM-Newton & MOS1 & PrimeFullWindow & Medium & 0113020101 & $2001-04-15$ & $7.3 \mathrm{ks}$ & $0.50 \pm 0.01$ \\
XMM-Newton & MOS2 & PrimeFullWindow & Medium & 0113020101 & $2001-04-15$ & $7.5 \mathrm{ks}$ & $0.52 \pm 0.01$ \\
XMM-Newton & PN & PrimeSmallWindow & Thin & 0113020101 & $2001-04-15$ & $16 \mathrm{ks}$ & $1.62 \pm 0.01$ \\
XMM-Newton & MOS1 & PrimeFullWindow & Thick & 0113020301 & $2001-11-08$ & $7.5 \mathrm{ks}$ & $0.45 \pm 0.01$ \\
XMM-Newton & MOS2 & PrimeFullWindow & Thick & 0113020301 & $2001-11-08$ & $6.2 \mathrm{ks}$ & $0.46 \pm 0.01$ \\
XMM-Newton & PN & PrimeSmallWindow & Thin & 0113020301 & $2001-11-08$ & $16 \mathrm{ks}$ & $1.63 \pm 0.01$ \\
\hline
\end{tabular}
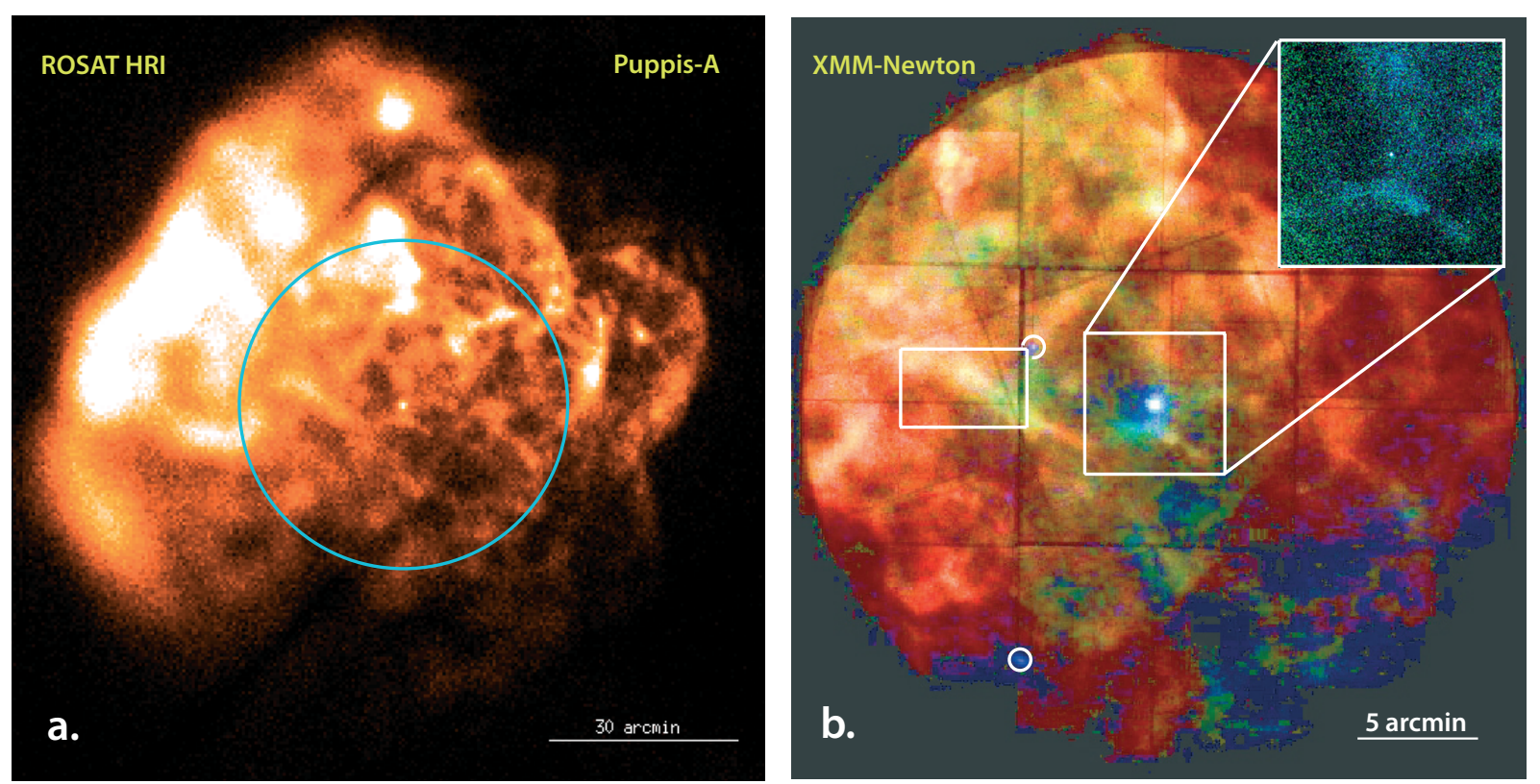

Fig. 1. a) Composite ROSAT HRI image of the Puppis-A supernova remnant. The blue ring indicates the 30 arcmin central region which has been observed by XMM-Newton in April and November 2001. b) XMM-Newton MOS1/2 false color image of the inner 30 arcmin central region of Puppis-A (red: $0.3-0.75 \mathrm{keV}$, green: $0.75-2 \mathrm{keV}$ and blue: $2-10 \mathrm{keV}$ ). The central source is RX J0822-4300. The inset shows the squared region as observed by the Chandra HRC-I. It is interesting to note that the region around RX J0822-4300 comprise mainly hard X-ray photons. The left white box indicates the region of the swirl-like structure interpreted by Winkler et al. (1989) as a second supernova in Puppis-A. The location of the northern and southern hard X-ray point sources are indicated by circles. The binning factors in the XMM and Chandra images are 4 arcsec and 0.6 arcsec, respectively. Adaptive smoothing with a Gaussian kernel of $\sigma<1$ pixel has been applied to the XMM-Newton and Chandra images. Top is north and left is east.

the algorithm used to determine the centroid of the charge cloud exiting the rear micro-channel plate of the HRC. Furthermore, aspect offset was corrected for the event files.

The ACIS observation was performed on 2000 January 1 using the front-illuminated (FI) ACIS-S2 chip with a frame time of $0.84 \mathrm{~s}$. RX J0822-4300 is located $\sim 2.7$ arcmin off-axis in this ACIS observation. In order to correct for possible pileup effects, we started our analysis again with level-1 files as those have preserved a number of source events which could have been misidentified as afterglows of cosmic ray events in the standard processing of level-2 data (cf. Davis 2002). The sub-arcsecond resolution of Chandra allows to extract the counts for the spectral analysis from a circle with radius 2.6 arcsec (encircled energy $\sim 99 \%$ for on-axis point sources). This selection radius minimizes the contamination from the supernova background emission. An annular region with radii between $\sim 2.6-5.3$ arcsec, centered at RX J0822-4300 was chosen to extract the background spectrum. Response files were created using the tools MKRMF and MKARF of CIAO. The background corrected HRC and
ACIS count rates of RX J0822-4300 are given in Col. 8 of Table 1.

From the ACIS-S2 and XMM-Newton data we found that the energy of the central source peaks at $\sim 1.5 \mathrm{keV}$. With the peak energy and for the off-axis angle of $\sim 0.3$ arcmin we extracted the desired point spread function (PSF) model images from CALDB 2.26 standard library files (F1) by interpolating within the energy and off-axis angle grids by using CIAO tool MKPSF. Exposure maps for the corresponding images were generated by the tool MKEXPMAP.

\section{Data analysis}

\subsection{Spatial analysis}

Composite images of the supernova remnant Puppis-A and its central region around RX J0822-4300, as seen by the ROSAT HRI, by XMM-Newton's MOS1/2 CCDs and by the Chandra HRC-I, are shown in Fig. 1. RX J0822-4300 is located at the center of these images. From Fig. $1 b$ it can be seen that 
the hardest X-ray emission in the remnant is mainly contributed by the central compact object RX J0822-4300. Apart from this, we observed two more hard X-ray sources in the XMM-Newton MOS1/2 images. Their locations as indicated by circles in Fig. $1 \mathrm{~b}$ are $\mathrm{RA}=08^{\mathrm{h}} 22^{\mathrm{m}} 26.70^{\mathrm{s}}$, Dec $=-43^{\circ} 10^{\prime} 25.99^{\prime \prime}(\mathrm{J} 2000)$ for the source located in the south and RA $=08^{\mathrm{h}} 22^{\mathrm{m}} 24.25^{\mathrm{s}}$, Dec $=-42^{\circ} 58^{\prime} 00.82^{\prime \prime}(\mathrm{J} 2000)$ for the northern source. The location of the northern source is close to the region which was suggested by Winkler et al. (1989) to be a second supernova within Puppis-A. These authors have observed an unusual swirl-like structure in optical images and interpreted this as a possible second supernova remnant. The center coordinate of this structure is at about RA $=08^{\mathrm{h}} 22^{\mathrm{m}} 39^{\mathrm{s}}$, Dec $=-42^{\circ} 59^{\prime} 41^{\prime \prime}(\mathrm{J} 2000)$. The left box in Fig. 1b illustrates the field of view in their observations. The angular separation between the northern hard X-ray source and the center of the swirl-like structure is $\sim 3.2$ arcmin. From the spectral analysis of optical filaments, Winkler et al. (1989) estimated that the kinematic age of the proposed second SNR is $<800$ years. If the northern hard X-ray source is correlated with this structure and this age estimate is correct it would require a space velocity $>2000 \mathrm{~km} \mathrm{~s}^{-1}$ (for an assumed distance of $2.2 \mathrm{kpc}$ ) in order to travel to its observed location. An association thus would be unlikely due to this high space velocity. For the southern hard X-ray source its correlation with Puppis-A is unspecified though most likely this is a background source. The photon statistics does not support a detailed spectral analysis for these two sources.

The XMM-Newton MOS1/2 false color image (Fig. 1b) demonstrates nicely that the south-western part of the remnant as well as the region near RX J0822-4300 comprise mainly hard X-ray photons. This is different from other parts of the image which consist of soft X-rays from the hot supernova ejecta. As Puppis-A is located at the edge of the Vela supernova remnant (distance $\sim 0.25 \mathrm{kpc}$ ), and is located behind it, we speculate that there is intervening absorbing material from Vela along the line of sight which absorbs most of the soft X-ray photons of the south-western part of Puppis-A. This view is supported by Fig. 2 which shows a belt of absorbing material crossing the whole Puppis-A supernova remnant from the south-western to the north-eastern direction (Aschenbach 1994, and discussion therein)

The high resolution X-ray image from HRC-I allows for the first time to examine the spatial nature of RX J0822-4300 with sub-arcsecond resolution. However, we have found that the full width half maximum ( $F W H M)$ of the point spread function (PSF) $(\sim 0.4$ arcsec) generated from the library files is narrower than expected. This can be ascribed to the fact that the PSF library files are derived by a ray-tracing program instead of obtained directly from the calibration data. Due to this caveat, it is legitimate to fit the image with the convolution of a 2-dimensional Gaussian function instead of a delta function. The radial profile of RX J0822-4300 is depicted in Fig. 4. The solid curve represents the best-fit Gaussian model with the modeled PSF at $1.5 \mathrm{keV}$ as a convolution kernel. The best-fit results yield a $F W H M$ of $0.59 \pm 0.01$ arcsec which is very close to the expected width of the Chandra PSF. This result appears as the first evidence for the point source emission nature of RX J0822-4300. Moreover, we were also able to narrow down the position of this compact object to the smallest region that has never been obtained before. The best-fit gives us a position for RX J0822-4300 which is RA $=08^{\mathrm{h}} 21^{\mathrm{m}} 57.40^{\mathrm{s}}$ and Dec $=$ $-43^{\circ} 00^{\prime} 16.69^{\prime \prime}(\mathrm{J} 2000)$. The statistical error of the position introduced by the image-fitting is found to be $0.01 \operatorname{arcsec}(1-\sigma)$. The predominant uncertainty of the source position is given by

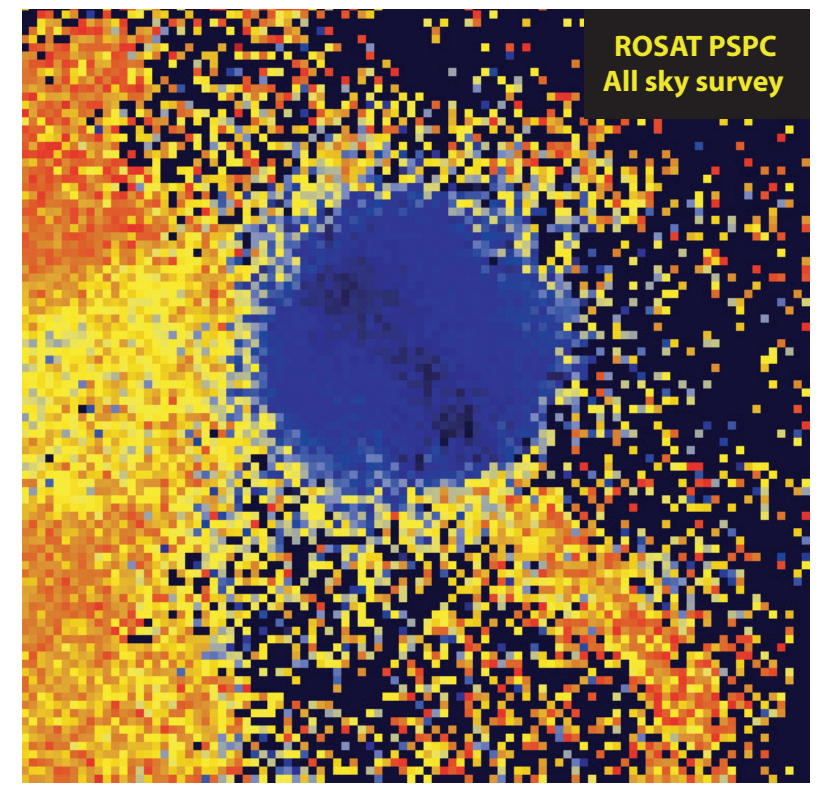

Fig. 2. False color image of Puppis-A and parts of the Vela supernova remnant as seen in the ROSAT all-sky survey. Puppis-A appears solely in blue color (harder X-rays) while the soft emission from the Vela supernova remnant is represented by the red and yellow colors. The image clearly demonstrates the existence of the absorption belt which crosses Puppis-A from the south-west to north-east direction and which is associated with rim emission from the Vela supernova remnant.
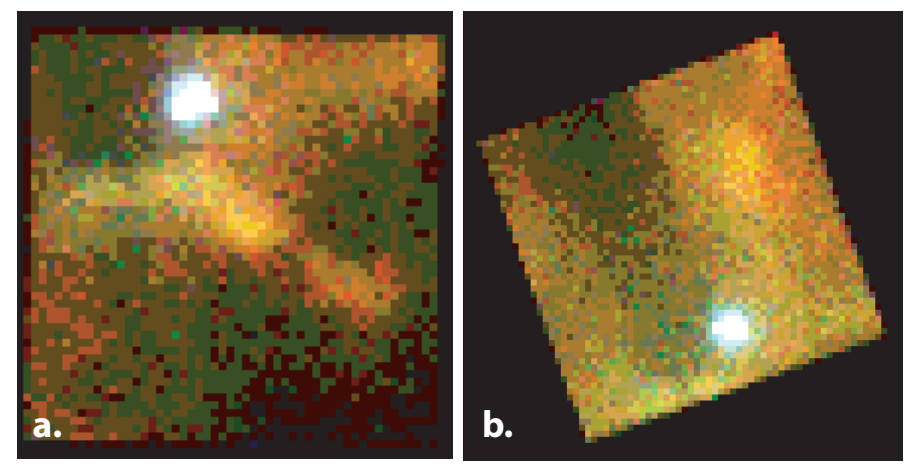

Fig. 3. False color image (red: $0.3-0.75 \mathrm{keV}$, green: $0.75-2 \mathrm{keV}$ and blue: $2-10 \mathrm{keV}$ ) of the $4.4^{\prime} \times 4.4^{\prime}$ region around RX J0822-4300 as seen by XMM-Newton's EPIC-PN detector during the observations in April 2001 a) and November 2001 b). The rim emission from Puppis-A near to the location of RX J0822-4300 is visible very well in a). Top is north and left is east.

the finite width of the PSF $(\sim 0.5$ arcsec $)$ and the average pointing accuracy of the satellite $(\sim 0.6 \mathrm{arcsec})$. The same position is obtained from the analysis of the HRC-S data. The position and point source character of RX J0822-4300, deduced by using Chandra, are in agreement with what we found in the XMM-Newton data (PSF $\sim 5 \operatorname{arcsec} F W H M$ ).

From observations of the neutral hydrogen surrounding RX J0822-4300, Reynoso et al. (2003) found a depression in the $\lambda 21-\mathrm{cm}$ line emission near to RX J0822-4300. According to their interpretation this structure could be connected to the compact stellar remnant because of its symmetric appearance as well as because of its alignment with the remnants optical expansion center and the position of RX J0822-4300. In order to search whether there is an X-ray structure near to RX J0822-4300 which correlates with this radio structure we have overlaid the radio contours from Reynoso et al. (2003) on the XMM-Newton 


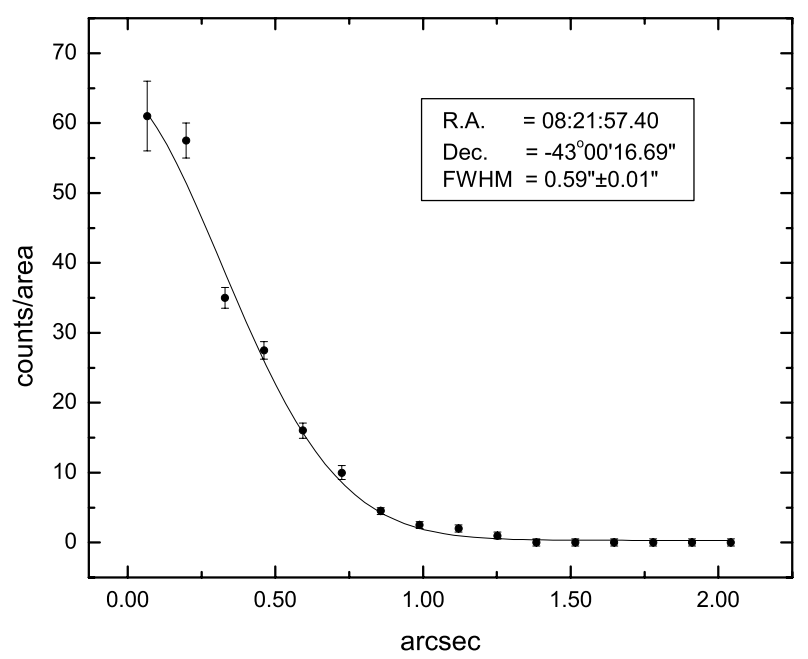

Fig. 4. Chandra HRC-I brightness profile for RX J0822-4300. The solid curve represents the best-fit Gaussian model with the modeled PSF at $1.5 \mathrm{keV}$ as a convolution kernel.
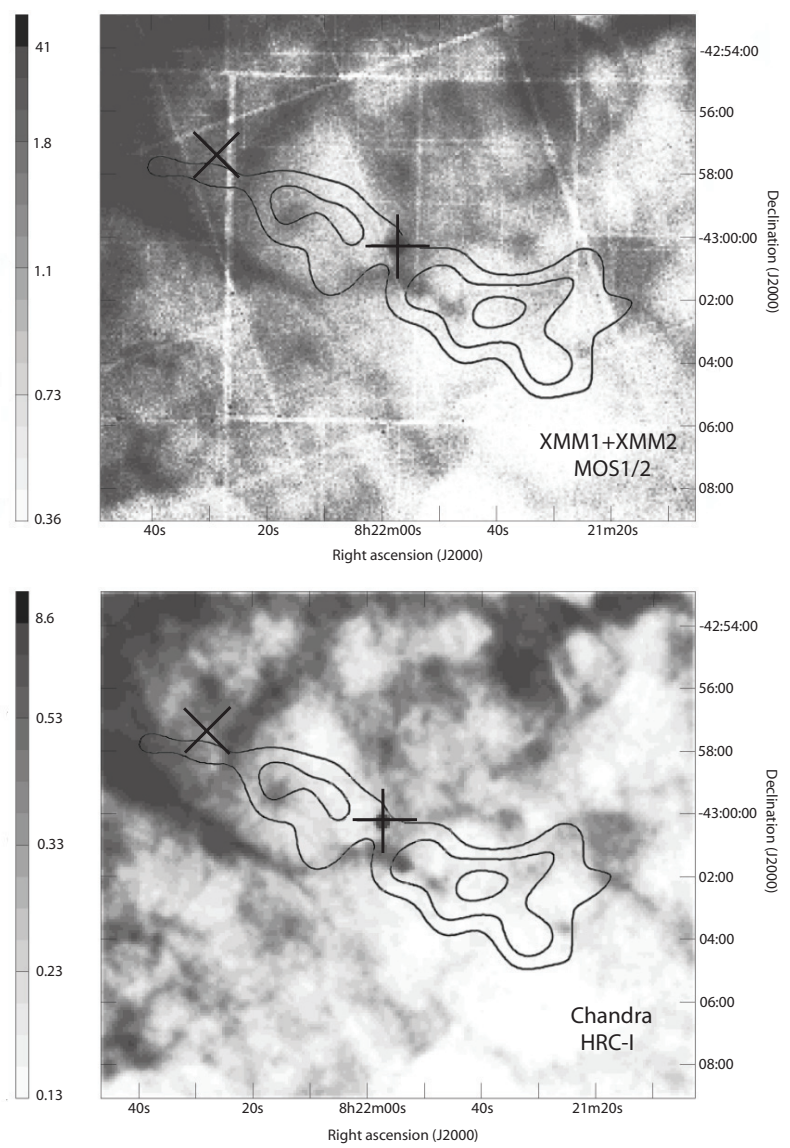

Fig. 5. The contours depict a double-lobed feature of reduced $\lambda 21-\mathrm{cm}$ line emission (from Reynoso et al. 2003) superimposed on fluxed image of XMM-Newton MOS1/2 (upper) and Chandra HRC-I (lower) in gray scale. The cross shows the optical expansion center of Puppis-A as calculated by Winkler et al. (1988).

and Chandra HRC-I image (cf. Fig. 5). No clear correlation between the radio and X-ray structures is seen, though the patchy supernova environment makes any conclusion uncertain.

\subsection{Spectral analysis}

We estimated the effects of pileup in both XMM1 and XMM2 data by using the XMMSAS task EPATPLOT. Our results showed that all the EPIC data were not affected by CCD pileup. Using the spectral parameters of RX J0822-4300 inferred from XMM-Newton, we estimated with the aid of PIMMS (version 3.6a) that the ACIS-S2 data are piled-up by a fraction of $\sim 11 \%$. We applied adequate correction by incorporating a pileup model in the spectral fitting (Davis 2001). Chandra data were also corrected for the degradation of quantum efficiency.

In order to constrain the spectral parameters tightly, we fitted XMM1, XMM2, as well as the ACIS-S2 data simultaneously. In order to obtain spectra from different observations and instruments with compatible significance, the energy channels were grouped dynamically with respect to the photon statistics in the analyzed data sets. For the MOS1/2 data of XMM2, we grouped the data to have at least 50 counts per bin. For the MOS1/2 data of XMM1 as well as the ACIS-S2 data we applied a grouping so as to have at least 100 counts per spectral bin. For the EPIC-PN data from XMM1 and XMM2 a grouping of $200 \mathrm{cts} / \mathrm{bin}$ was used. All spectral fits were performed in the 0.3-10 keV energy range by using XSPEC 11.3.1.

Various model spectra like single blackbody, double blackbody, power-law, combinations of blackbody and power-law, broken power-law, as well as thermal bremsstrahlung were fitted to the data. Independent of the fitted spectral models we found that the fits improve if the spectrum extracted from the Chandra data was not included in the analysis. Since the spectra extracted from XMM-Newton data supersedes the Chandra data in photon statistics, we excluded the later from the spectral analysis without loss of generality. The parameters of all fitted model spectra are summarized in Table 2. The quoted errors are conservative and are $1 \sigma$ for 2 parameters of interest for single component spectral models and for 3 parameters of interest for multi-component model.

Fitting the spectral parameters of RX J0822-4300 as inferred from ROSAT PSPC data by PBW96 we found that these parameters $\left(T=3.2 \times 10^{6} \mathrm{~K}, N_{\mathrm{H}}=4.1 \times 10^{21} \mathrm{~cm}^{-2}, R=2 \mathrm{~km}\right)$ yield no acceptable description of the XMM-Newton observed spectrum $\left(\chi_{v}^{2}=21.17\right.$ for 467 d.o.f. $)$. In general, spectral fitting with a single component blackbody or power-law model did not model the data beyond $\sim 3 \mathrm{keV}$ (cf. Table 2). Testing multi-component models we found that a two component blackbody with $N_{\mathrm{H}}=4.54_{-0.43}^{+0.49} \times 10^{21} \mathrm{~cm}^{-2}, T_{1}=2.61_{-0.26}^{+0.30} \times 10^{6} \mathrm{~K}$, $T_{2}=5.04_{-0.20}^{+0.28} \times 10^{6} \mathrm{~K}$ and $R_{1}=3.29_{-0.74}^{+1.12} \mathrm{~km}, R_{2}=0.75_{-0.15}^{+0.12} \mathrm{~km}$ for the blackbody temperatures and emitting areas, respectively, yields the best description of the observed spectrum. The reduced- $\chi^{2}$ of this fit is 1.20 for 465 d.o.f. We note that the apparent deviation of the reduced- $\chi^{2}$ from one, indicating an acceptable fit, is due to the fact that the data from different instruments and different epochs are modeled simultaneously. The benefit of combining all spectral data in simultaneous fits is the higher photon statistics and thus the ability to better discriminate between competing model spectra.

Figures 6 and 7 shows the spectral fit for an absorbed double blackbody model and the corresponding spectral components respectively. In order to properly constraint the parameter space for the best-fitting model, we calculated the contour plots in the $T_{1}-R_{1}, T_{1}-N_{\mathrm{H}}$ and $T_{2}-R_{2}, T_{2}-N_{\mathrm{H}}$ planes, respectively. These plots are depicted in Fig. 8. For a consistence check we modeled the Chandra ACIS-S2 spectrum with the best fitting double blackbody model and found all parameters in agreement with those fitted for the XMM-data. 


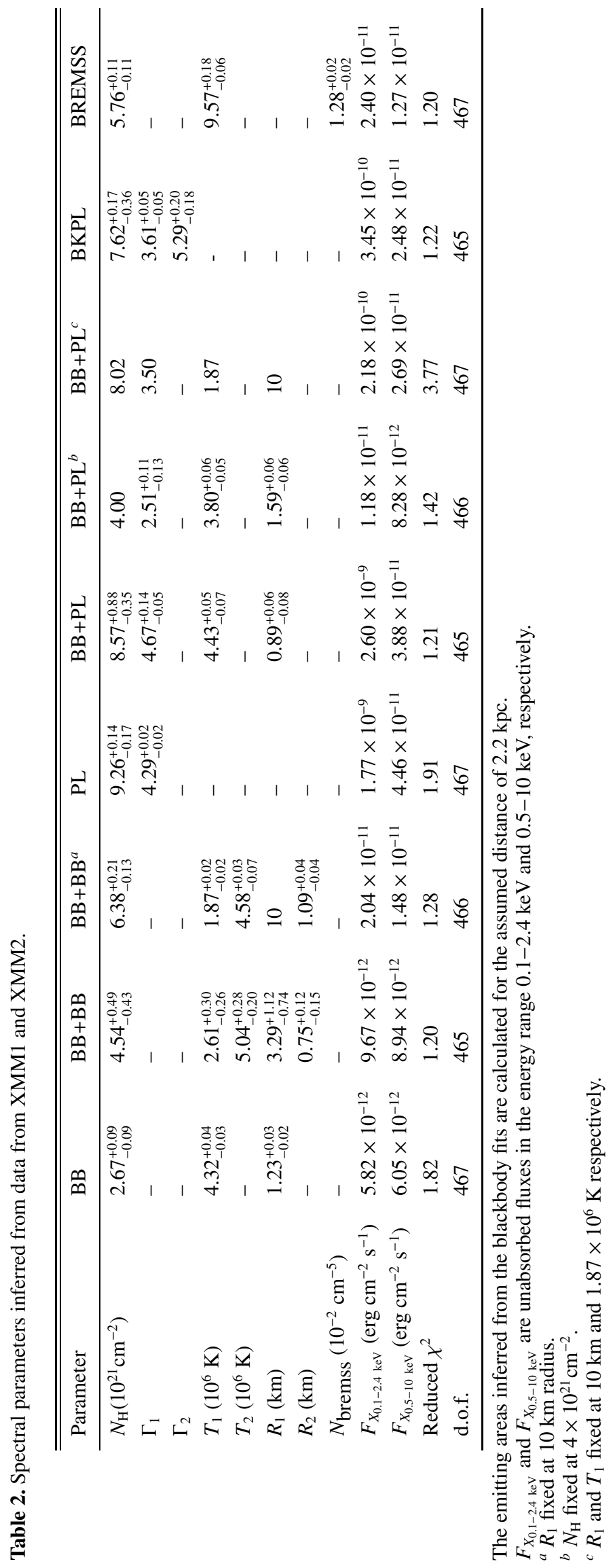

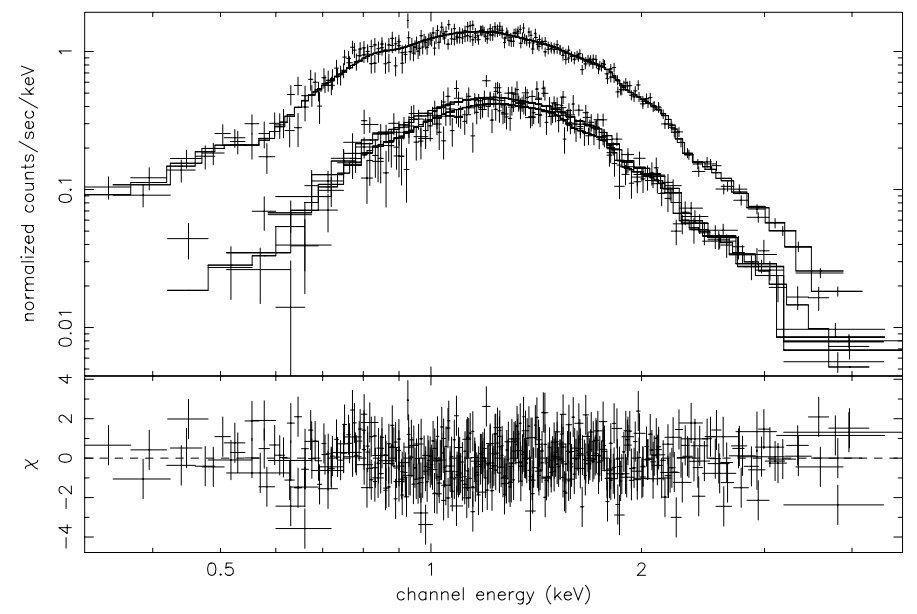

Fig. 6. Energy spectrum of RX J0822-4300 as observed with the EPICPN (upper spectra) and MOS $1 / 2$ detectors (lower spectra) and simultaneously fitted to an absorbed two component blackbody model (upper panel) and contribution to the $\chi^{2}$ fit statistic (lower panel).

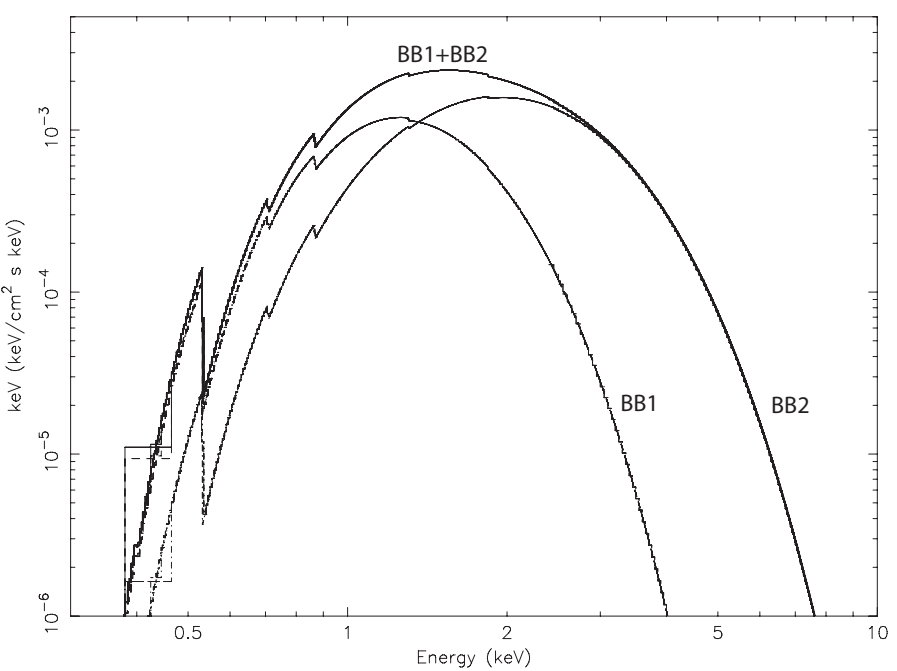

Fig. 7. Components and combined model of double blackbody fitted to the spectral data of RX J0822-4300.

Both $R_{1}$ and $R_{2}$ inferred from the double blackbody fit are inconsistent with the size of a canonical neutron star (i.e. $R$ $10 \mathrm{~km})$. It is therefore instructive to redo the fitting for this model with $R_{1}$ fixed at $10 \mathrm{~km}$. This model still yields acceptable values of $N_{\mathrm{H}}=6.38_{-0.13}^{+0.21} \times 10^{21} \mathrm{~cm}^{-2}, T_{1}=1.87_{-0.02}^{+0.02} \times$ $10^{6} \mathrm{~K}, T_{2}=4.58_{-0.07}^{+0.03} \times 10^{6} \mathrm{~K}$, and $R_{2}=1.09_{-0.04}^{+0.04} \mathrm{~km}$ with $\chi_{v}^{2}=1.28$ (for 466 d.o.f.) only a slightly larger than leaving $R_{1}$ unconstrained.

For a model combining a blackbody and a power-law, the goodness-of-fit is compatible with that for the double blackbody model $\left(\chi_{v}^{2}=1.21\right.$ for 465 d.o.f.). The inferred slope of the power-law component is $\Gamma=4.67_{-0.05}^{+0.14}$. Although this is steeper than the photon-index, $\Gamma=1-3$, observed for rotationpowered pulsars (cf. Becker \& Trümper 1997), the model cannot be rejected simply based on this as it is not a priori applicable for central compact objects. However, the column density is much higher than the expected level. When $N_{\mathrm{H}}$ is fixed to $4 \times 10^{21} \mathrm{~cm}^{-2}$, which is consistent with the values obtained by PBW96, ZTP99 and Winkler et al. (1981), it results in a parameter set of $\Gamma=2.51_{-0.13}^{+0.11}, T=3.80_{-0.05}^{+0.06} \times 10^{6} \mathrm{~K}, R=1.59_{-0.06}^{+0.06} \mathrm{~km}$, though with a large $\chi_{v}^{2}$ of 1.42 for 466 d.o.f. 

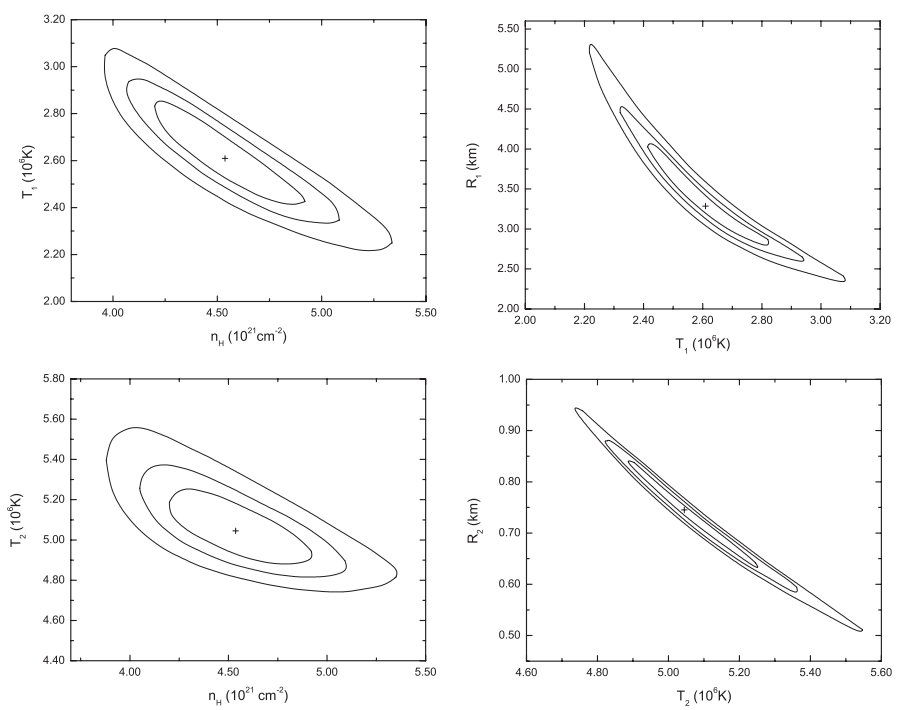

Fig. 8. $1 \sigma, 2 \sigma$ and $3 \sigma$ confidence contours for the double blackbody fit to the X-ray spectrum of RX J0822-4300.

It is necessary to examine whether a broken power law model can describe the spectra. This implies a purely non-thermal emission with spectral steepening at high energy after an energy break which is due to the deficit of energetic emitting charged particles. From Table 2 it is obvious that the broken power law model does not yield any photon index that is consistent with that of a typical pulsar. We also fitted the data with a thermal bremsstrahlung model which physically implies that the central compact object would be surrounded by a hot plasma. From the normalization constant inferred from the spectral analysis, we can calculate the extent of the plasma. Following Iaria et al. (2001), we assume the bremsstrahlung normalization to be $N_{\text {bremss }}=3.02 \times 10^{-15} N_{\mathrm{e}}^{2} V / 4 \pi D^{2}$, where $D$ is the distance to the source in $\mathrm{cm}, N_{\mathrm{e}}$ is the electron density $\left(\mathrm{cm}^{-3}\right)$, and $V$ is the volume of the bremsstrahlung emitting region. Assuming $N_{\mathrm{e}}$ is comparable with the average density $\sim 1 \mathrm{~cm}^{-3}$ of Puppis-A (Petre et al. 1982), the radius of the assumed spherical emitting region is estimated to be $\sim 2.7 \mathrm{pc}$ for an adopted distance of $2.2 \mathrm{kpc}$. This implies that the source should be extended (at a level of $\sim 4.2$ arcmin assuming a distance of $2.2 \mathrm{kpc}$ ), in contradiction to the results from the spatial analysis.

From both, the XMM-Newton MOS1/2 and the Chandra HRC-I images, some faint and diffuse hard X-ray emission around RX J0822-4300 seems to be present (cf. Figs. 1 and 3 ). Its nature can be determined by examining its spectrum. We extracted the events in the X-ray filament near to RX J0822-4300 from the MOS1/2 cameras of XMM1 from a $80 \operatorname{arcsec} \times 30$ arcsec box centered at RA $=08^{\mathrm{h}} 21^{\mathrm{m}} 57.077^{\mathrm{s}}$, Dec $=-43^{\circ} 01^{\prime} 15^{\prime \prime} .42(\mathrm{~J} 2000)$. We found that its spectrum is consistent with an absorbed non-equlibrium ionization collisional plasma model (XSPEC model: VNEI) with goodnessof-fit of $\chi^{2}=151.08$ for 142 d.o.f. The energy spectrum as fitted to this model spectrum is displayed in Fig. 9. Line emission is easily recognized in this plot. The most obvious feature is the O VII and O VIII line complex at $0.662 \mathrm{keV}$ and $0.651 \mathrm{keV}$ respectively. Parameters inferred from the bestfitted model are the column density $N_{\mathrm{H}}=3.70_{-0.12}^{+0.12} \times 10^{21} \mathrm{~cm}^{-2}$, the plasma temperature $T=7.62_{-0.21}^{+0.10} \times 10^{6} \mathrm{~K}$, the ionization timescale $\tau=2.33_{-0.11}^{+0.15} \times 10^{11} \mathrm{~s} \mathrm{~cm}^{-3}$, and the metal abundances with respect to the solar values $\left(\mathrm{O}: 17.83_{-1.56}^{+1.58}, \mathrm{Ne}: 4.00_{-0.68}^{+0.67}\right.$, Si: $2.25_{-1.32}^{+1.33}$, S: $6.58_{-4.81}^{+4.85}$, Fe: $2.24_{-0.21}^{+0.20}$ ) (quoted errors are $1 \sigma$ for

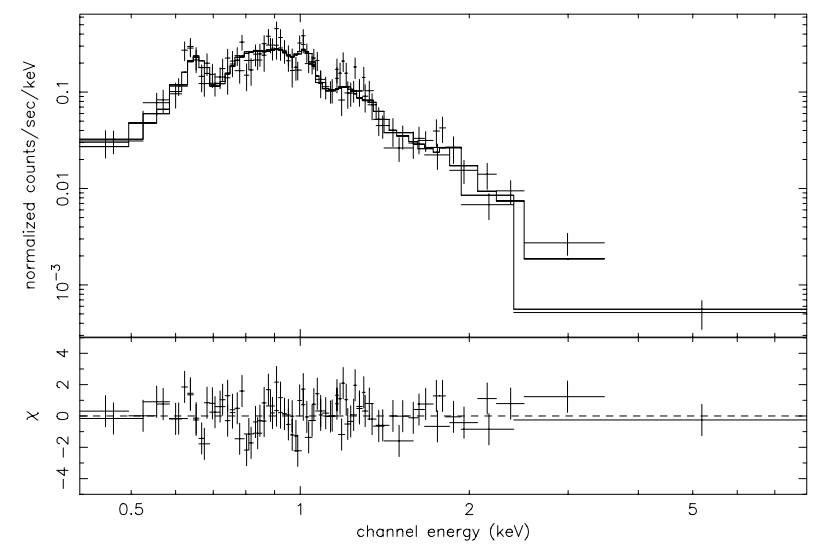

Fig. 9. Energy spectrum of the rim emission underneath RX J0822-4300 as observed in April 2001 with the EPIC-MOS1/2 detector and simultaneously fitted to an absorbed non-equilibrium ionization collisional plasma model (upper panel) and contribution to the $\chi^{2}$ fit statistic (lower panel).

2 parameters of interest). These parameters imply a relative abundance ratio O:Fe to be about 6-9 times its solar value which strongly suggest an enhancement of oxygen in Puppis-A. This is in agreement with the conclusion drawn by Canizares \& Winkler (1981). For the other elements, including $\mathrm{H}, \mathrm{He}, \mathrm{C}, \mathrm{Mg}, \mathrm{Ar}$, $\mathrm{Ca}$ and $\mathrm{Ni}$, we do not find any sign of enhancement and their abundances are in agreement with the solar values. We have performed the spectral fitting with different selected backgrounds. Provide that the backgrounds are selected from low count regions, all the best-fitted values are found to be within the quote $1 \sigma$ errors above. Since the remnant environment is patchy and inhomogeneous, abundance ratios from different regions are not expected to be comparable. A detailed modeling of the variation of chemical abundance is beyond the scope in this paper. A further detailed analysis of Puppis-A is in preparation and will be published elsewhere.

Although the rim emission appears to be a part of the structure of Puppis-A, we also exam whether there is any non-thermal contribution in the emission by adding a Crablike power-law component (i.e. with photon index of 2) in the spectral fit. The additional component does not improve the goodness-of-fit at all ( $\chi^{2}=150.95$ for 141 d.o.f.). A $3 \sigma$ upper bound of the power-law model normalization is estimated to be $6 \times 10^{-5}$ photons $\mathrm{keV}^{-1} \mathrm{~cm}^{-2} \mathrm{~s}^{-1}$. This implies a limiting flux of non-thermal plerionic emission, if any, to be $3.06 \times$ $10^{-13} \mathrm{erg} \mathrm{cm}^{-2} \mathrm{~s}^{-1}$ and $2.88 \times 10^{-13} \mathrm{erg} \mathrm{cm}^{-2} \mathrm{~s}^{-1}$ in $0.1-2.4 \mathrm{keV}$ and $0.5-10 \mathrm{keV}$ respectively.

\subsection{Timing analysis}

\subsubsection{Search for long-term variabilities}

To check whether the energy fluxes measured from RX J0822-4300 by XMM-Newton and Chandra are consistent with each other or whether there are significant long-term deviations observed in the different data sets we computed the flux for the best fitting double blackbody model from all available data. In order to compare the XMM-Newton and Chandra results with the existing ROSAT flux we restricted this computation to the energy range $0.1-2.4 \mathrm{keV}$. As shown in Table 3, all observed energy fluxes, from ROSAT to XMM-Newton are consistent with a constant energy flux of $f_{x}(0.1-2.4 \mathrm{keV}) \sim 3 \times 10^{-12} \mathrm{erg} \mathrm{cm}^{-2} \mathrm{~s}^{-1}$. The same conclusion 
Table 3. Observed fluxes inferred from observations at different epochs.

\begin{tabular}{lccc}
\hline \hline Observations & $\begin{array}{c}F_{X}(0.1-2.4 \mathrm{keV}) \\
\mathrm{ergs} \mathrm{cm}^{-2} \mathrm{~s}^{-1}\end{array}$ & $\begin{array}{c}F_{X}(0.5-10 \mathrm{keV}) \\
\mathrm{ergs} \mathrm{cm}^{-2} \mathrm{~s}^{-1}\end{array}$ & Start Date \\
\hline XMM1 & $3.37_{-0.26}^{+0.28} \times 10^{-12}$ & $4.19_{-0.36}^{+0.39} \times 10^{-12}$ & $2001-04-15$ \\
XMM2 & $3.38_{-0.28}^{+0.30} \times 10^{-12}$ & $4.17_{-0.37}^{+0.42} \times 10^{-12}$ & $2001-11-08$ \\
Chandra ACIS & $3.34_{-0.36}^{+0.40} \times 10^{-12}$ & $4.18_{-0.50}^{+0.54} \times 10^{-12}$ & $2000-01-01$ \\
ROSAT PSPC $^{\text {a }}$ & $\sim 3 \times 10^{-12}$ & - & $1991-04-16$
\end{tabular}

${ }^{a}$ Flux observed by ROSAT according to PBW96.

can be drawn from comparing the broadband fluxes from Chandra, XMM1 and XMM2. The observed flux of these three observations are found to be $\sim 4 \times 10^{-12} \mathrm{erg} \mathrm{cm}^{-2} \mathrm{~s}^{-1}$ in the range of $0.5-10 \mathrm{keV}$.

\subsubsection{Search for coherent pulsations}

Although the lack of long-term variability and the spectral analysis eliminates some highly improbable models and hence helps us to put constraints on the properties of the central compact object, the most strong argument that this object is indeed a neutron star would come from the detection of X-ray pulsations. Since the small-window mode was setup for the EPIC-PN camera in both XMM1 and XMM2 observations, the $5.7 \mathrm{~ms}$ temporal resolution of this data is sufficient to search for coherent short-term pulsations.

The arrival times in both event files were barycentric corrected using the XMMSAS task BARYCEN. In order to minimize the systematic errors induced in the barycentric correction, we use the position inferred from the Chandra HRC-I image fitting (cf. Sect. 3.1) for correcting the arrival times in both data sets. The initial period searches were performed by applying a fast Fourier transformation (FFTs) on both sets of photon arrival times separately. The advantage of having multiple data sets from different epochs supports to cross-check any periodicity candidates easily and prevents wrong identifications. FFTs were calculated for each time series with 20 different binnings. Searches in the frequency domain were limited at $0.01 \mathrm{~Hz} \leq$ $f \leq 100 \mathrm{~Hz}$. Promising frequency peaks appearing in the power spectra of both observations were cross-correlated and selected for subsequent searches using standard epoch-folding analysis.

PZT99 reported the detection of a periodic signal with $P=$ $75.2797300 \mathrm{~ms}$ and $\dot{P}=1.49 \times 10^{-13} \mathrm{~s} \mathrm{~s}^{-1}$ in their ROSAT analysis. We searched for coherent pulsations in a period range extrapolated to these spin parameters in XMM-Newton data. The photon statistics of this data, compared to the ROSAT data, is about a factor 25 higher. A similar analysis as reported in the present work was already performed by Becker \& Aschenbach (2002) who could not confirm the existence of a pulsed signal in an extrapolated period range, neither in the ROSAT nor in the XMM-Newton data. We herewith fully confirm their result in our independent analysis.

Taking peaks in the power spectra as initial candidates, we made a more detailed search using $Z_{m}^{2}$ test where $m$ is the numbers of harmonics (Buccheri et al. 1983). We have detected periodicities of $P=0.218749 \pm 0.000001 \mathrm{~s}$ in XMM1 (MJD 52014.4634390827268 days) $^{2}$ and $P=0.222527 \pm$ $0.000002 \mathrm{~s}$ in XMM2 (MJD 52221.8938398198225 days) which both have very similar properties. The quoted uncertainties

${ }^{2}$ The mean epoch of the observation in TDB at the solar system barycenter (SSB).
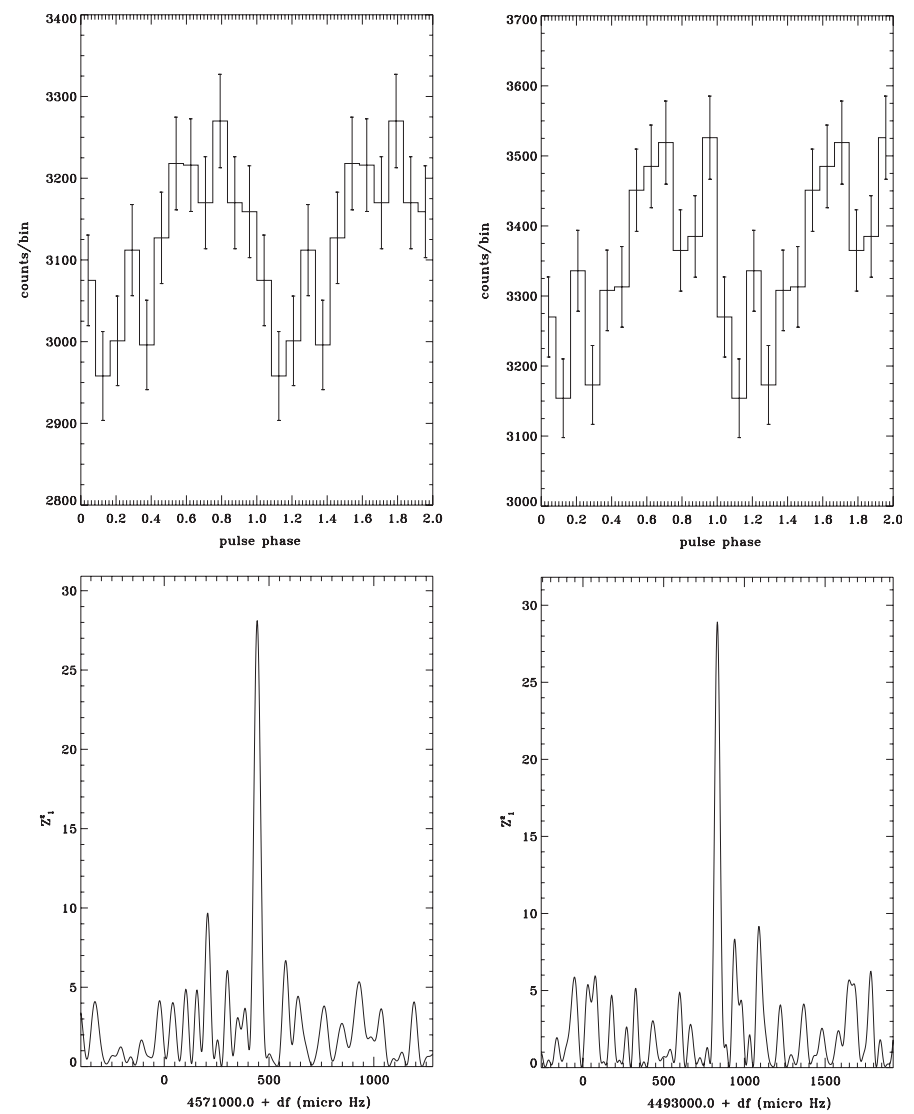

Fig. 10. Pulse profiles and $Z_{1}^{2}$-distribution of the folded light curves as found in the XMM-Newton EPIC-PN data taken in April (left panel) and November 2001 (right panel).

indicate the Fourier resolution $P^{2} / T$ in the corresponding observation, where $T$ is the time span in the data set. Using the $H$-test (De Jager et al. 1989), we found that $H$ is maximized for the first harmonic. The calculated $Z_{1}^{2}$ for the detected signals in XMM1 and XMM2 are 28.10 and 28.92, respectively. The nominal probabilities for the identification of these signals by chance are $8 \times 10^{-7}$ and $5 \times 10^{-7}$, respectively. The pulse profiles and $Z_{1}^{2}$ distribution is given in Fig. 10. Both lightcurves are similar to each other and share the same sinusoidal characteristics. Following Becker \& Trümper (1999), we calculated the pulsed fraction of this signal by the bootstrap method proposed by Swanepoel et al. (1996) and obtained $P_{\mathrm{f}}=5 \pm 1 \%$ in both, the XMM1 and XMM2 data sets. The period time derivative calculated from the separation of the epochs of the two data sets is $\dot{P}=(2.112 \pm 0.002) \times 10^{-10} \mathrm{~s} \mathrm{~s}^{-1}$.

Taking the number of $10^{5}$ trials into account, the probabilities for finding these signals by chance is $(5-8) \times 10^{-2}$. However, given the similarity in period, pulse shape, signal strength and pulse fraction together with the detection of the signal in two independent XMM-Newton data sets makes this signal a very promising candidate to test and search for in future observations.

In order to minimize the probability of a false detection we have investigated the possibility that the signals are induced from the readout processes in the detector CCDs or other cyclic processes operating during data acquisition. For this we have extracted events from Puppis-A in both, the XMM1 and XMM2 data sets from a location near to RX J0822-4300, i.e. from CCD columns located at the same level in the readout direction as RX J0822-4300. The same procedure of timing analysis was applied on these events as applied to the events 
from RX J0822-4300. However, we did not detect any cyclic signal at a period near to $\sim 0.22 \mathrm{~s}$. We therefore can rule out that the detected pulsations are due to periodic systematics in the onboard data processing during data acquisition.

To further cross-check this periodicity detection, we utilized an independent data set from Chandra HRC-S. Since the observation with $\mathrm{HRC}-\mathrm{S}$ was performed in "imaging" mode where the outer segments of the micro-channel plate were disabled, the total count rate is below the telemetry saturation limit, so that all events can be assigned with accurate time and the HRC timing anomalies are minimized. This enables us to perform an accurate timing analysis on this data set, though the photon statistics is a factor of $\sim 6$ lower than in the XMM-Newton data. The event file of HRC-S was firstly barycentric corrected (with the position given in the HRC-I image fit) by CIAO tool AXBARY. With the $P$ and $\dot{P}$ estimated from XMM-Newton data, we extrapolated the period to the epoch of HRC-S observation as an initial starting point. A detailed search around this period gives a promising candidate at $P=0.217303 \pm 0.000002 \mathrm{~s}$ in the Chandra HRC-S data (MJD 51934.6266560833901 days). The $H$-test indicates the highest probability for $Z_{7}^{2}=45.94$ wich yields a nominal chance probability of $3 \times 10^{-5}$. This is not strong enough to conclude a significant signal in the HRC-S data though we point out that there are only $\sim 6000$ counts available for this test. For a 5\% pulsed fraction as indicated in the XMM-Newton data only $\sim 300$ counts would contribute to the pulsed component. The low significance of the signal found in the Chandra data thus would be in line with a low significance of the periodic signal. Archival ROSAT and ASCA data of RX J0822-4300 are of small photon statistics so that we did not include this data in order to search for a pulsed signal near to $0.22 \mathrm{~s}$.

\section{Discussion and conclusion}

The lack of any detectable long term variability together with the high X-ray/optical flux ratio and the observed spectral characteristics makes it very unlikely that RX J0822-4300 is something else but the compact stellar remnant formed in the core collapsed supernova which was left behind Puppis-A. Our image analysis shows that the compact object is the hardest X-ray source in the 30 arcmin central region of Puppis-A. Chandra and XMM-Newton data do not show any extended X-ray emission which could be plerionic emission powered by the compact remnant, though this is difficult to quantify given the patchy environment in which RX J0822-4300 is located.

From the spectral fitting, we found that the point-source spectrum is compatible with a two component blackbody model. The best-fit model yields $N_{\mathrm{H}}=4.54_{-0.43}^{+0.49} \times 10^{21} \mathrm{~cm}^{-2}$, temperatures of $T_{1}=2.61_{-0.26}^{+0.30} \times 10^{6} \mathrm{~K}$ and $T_{2}=5.04_{-0.20}^{+0.28} \times 10^{6} \mathrm{~K}$ for the projected blackbody emitting areas with radii $R_{1}=3.29_{-0.74}^{+1.12} \mathrm{~km}$ and $R_{2}=0.75_{-0.15}^{+0.12} \mathrm{~km}$, respectively. Compared to standard cooling curves (e.g. Yakovlev et al. 2004) $T_{1}$ is a little higher than $\sim 1.8 \times 10^{6} \mathrm{~K}$ which would be expected for a $1.35 M_{\odot}$ neutron star with a stiff equation of state (Prakash et al. 1988). $R_{1}$ is not quite consistent with the size of a typical neutron star. This was already found by ZTP99 who attempted to obtain a set of reasonable neutron star parameters by modeling the ROSAT data with spectral models which take the presence of a hydrogen atmosphere into account, though the applicability of these models are restricted.

In the present work we have shown that a parameter set which is consistent with a standard cooling neutron star model can be obtained by fixing $R_{1}$ at $10 \mathrm{~km}$. This yields a column density of $6.38_{-0.13}^{+0.21} \times 10^{21} \mathrm{~cm}^{-2}$, temperatures of $T_{1}=1.87_{-0.02}^{+0.02} \times$ $10^{6} \mathrm{~K}$ and $T_{2}=4.58_{-0.07}^{+0.03} \times 10^{6} \mathrm{~K}$ for a projected blackbody emitting area with radius $R_{2}=1.09_{-0.04}^{+0.04} \mathrm{~km}$.

The results of our analysis suggest that the low temperature component is emitted from a large fraction of the neutron star surface, while the high temperature component is emitted from a much smaller and hotter region. This double blackbody model, though, could be a two-step adaption for a wider temperature distribution which centrally peaks. Such a hot spot on the neutron star surface can be produced by several mechanisms. One of them is the bombardment of the polar cap regions by energetic particles accelerated in the magnetosphere backwards to the neutron star surface (Cheng et al. 1986; Cheng \& Zhang 1999). Another way to produce a hot spot on the stellar surface is by anisotropic heat transport (Page 1995). Since the heat conduction inside a neutron star is much more efficient in the direction along the magnetic field lines than that in the perpendicular direction, a complete model of cooling magnetic neutron star should lead to an anisotropic heat flow and hence produce hot spots on the stellar surface. In this scenario, one should expect the emission to be pulsed at the rotation period of the star as the hot spot goes across the line of sight. A pulsed X-ray flux as revealed by the putative periodic signals seen in XMM1 and XMM2 thus would support this scenario.

The pulsed fraction of the putative periodic signal is $5 \pm$ $1 \%$ in XMM1 and XMM2. The emission from young pulsars like the Crab is compatible with being $100 \%$ pulsed (Tennant et al. 2001), whereas the fraction of pulsed photons is $\sim 7 \%$ for the Vela pulsar and $\sim 20-40 \%$ in many of the other X-ray detected pulsars (e.g. Becker \& Pavlov 2001; Becker \& Aschenbach 2002; Kaspi et al. 2004). A low pulsed fraction, however, is not unexpected though. When the general relativistic effect is taken into account (Page 1995; Hui \& Cheng 2004), the pulsations are found to be strongly suppressed and the pulsed fraction is highly dependent on the mass to radius ratio of the star, the orientation of the hot spot and the viewing angle geometry. This is due to the fact that the gravitational bending of light will make more than half of the stellar surface become visible at any instant and hence the contribution of the hot spot will be hampered. If the orientation of the hot spot is deviated from that of an orthogonal rotator and/or the star has a high mass to radius ratio, then a very low amplitude pulsations is expected, which makes the periodicity search difficult.

The $\dot{P}$ deduced for the candidate periodicity would be among the largest spin-down rates in the neutron star population. The largest known $\dot{P}$ was inferred from SGR 1806-20, $\dot{P}=(8-47) \times 10^{-11} \mathrm{~s} \mathrm{~s}^{-1}$, (Kouveliotou et al. 1998; Woods et al. 2002). If the identifications of $P$ and $\dot{P}$ are correct, it implies a non-steady spin-down behavior of RX J0822-4300. This phenomenon is not unobserved. There are two SGRs (SGR 1806-20 and SGR 1900+14) which show large changes in the spin-down torque up to a factor of $\sim 4$ (Woods et al. 2002). Moreover, deviations from a steady spin-down were also observed in the other radio-quiet neutron stars such as in SNR PKS 1209-51/52 (Zavlin et al. 2004). However, the rotational dynamics cannot be determined without ambiguity here and further observations are needed to confirm and/or refine this putative periodicity.

An alternative proposal to explain the origin of X-rays from radio quiet compact objects in supernova remnants is accretion onto a neutron star (e.g. Pavlov et al. 2000). In this scenario, the observed luminosity $L$ is powered by an accretion rate of $\dot{M}=L /\left(\zeta c^{2}\right)$. $\zeta$ is the accretion efficiency which is expressed as $\zeta=0.2 M_{1.4} R_{6}^{-1}$. Equating the expression of $\dot{M}$ 
with Bondi formula (i.e. $\dot{M}=4 \pi G^{2} M^{2} \rho v^{-3}$ ), we can express the relation of the circumstellar baryon density as $n=8 \times$ $10^{3} v_{100}^{3}(0.2 / \zeta) M_{1.4}^{-2} L_{33} \mathrm{~cm}^{-3}$, where $v_{100}$ is the velocity of the neutron star in the unit of $100 \mathrm{~km} \mathrm{~s}^{-1}$ and $L_{33}$ is the luminosity in the unit of $10^{33} \mathrm{erg} \mathrm{s}^{-1}$. The offset of $\sim 6.1 \mathrm{arcmin}$ from the optical expansion center, estimated distance $(\sim 2.2 \mathrm{kpc})$ and estimated age $(\sim 3700$ years $)$ suggest a transverse velocity of $\sim 985 \mathrm{~km} \mathrm{~s}^{-1}$. Even we take $L_{33}=1$, which is lower than the value inferred from the acceptable spectral fit $L_{33} \sim 5$, the expression of $n$ implies a density of about 6 orders of magnitude higher than the expected value found by Petre et al. (1982).

It is therefore safe to reject the scenario that the observed $\mathrm{X}$-rays are powered by accretion from circumstellar matter. The stringent optical limit also rules out the possibility that the accretion is from a massive companion. However, we cannot completely exclude the possibilities that the central object is accreting from a very close dwarf star or from a fossil disk (van Paradijs et al. 1995) which remained after the supernova explosion. For the first possibility, even though such a compact system is unlikely to remain bound in the disruption of the high mass progenitor ( $225 M_{\odot}$ Canizares \& Winkler 1981), a deeper search for optical/IR counterpart can still be beneficial.

As has been shown in this work, the properties of the compact object in Puppis-A are similar to those of other radio quiet neutron stars in many aspects (e.g. Cas A; Pavlov et al. 2000; Chakrabarty et al. 2001; Murray et al. 2002). Even though the nature of this class of object has not yet been completely resolved, their existence has already revolutionized the conventional notion of neutron stars and their environment. Since it is easier to detect and identify active radio/X-ray pulsars than the radio-quiet neutron stars which are only observable in X-ray and located in a patchy X-ray bright supernova remnant, it is plausible that they are more common than canonical pulsars. It is not unlikely to assume that the relatively small number of detected radio-quiet neutron stars is due to observational selection effects. In this sense, identifying the nature of this class, including RX J0822-4300, is very important.

Acknowledgements. We acknowledge discussion with Bernd Aschenbach and thank Jürgen Fath and our colleagues at MPE for their support. We also thank the referee for thoroughly reading the manuscript and provide us with many useful comments.

\section{References}

Aschenbach, B. 1994, in New Horizon of X-ray Astronomy - First Results from ASCA, ed. F. Makino, \& T. Ohashi, Frontier Science Series No. 12, 103 Aschenbach, B. 1998, Nature, 396, 141

Becker, W., \& Trümper, J. 1997, A\&A, 326, 682

Becker, W., \& Trümper, J. 1999, A\&A, 341, 803

Becker, W., \& Pavlov, G. G. 2001, in The Century of Space Science, ed. J. Bleeker, J. Geiss, \& M. Huber (Kluwer Academic Publishers), 721 [arXiv: astro-ph/0208356]

Becker, W., \& Aschenbach, B. 2002, in Proceedings of the WE-Heraeus Seminar on Neutron Stars, Pulsars and Supernova remnants, ed. W. Becker, H. Lesch, \& J. Trümper, MPE-Report, 278, 64 [arXiv: astro-ph/0208466]

Brazier, K. T. S., \& Johnston, S. 1999, MNRAS, 305, 671

Buccheri, R., et al. 1983, A\&A, 128, 245

Burke, B. E., et al. 1997, IEEE Trans. Elec. Dev., 44, 1633

Camilo, F. 2003, in Radio Pulsars, ed. M. Bailes, D. J. Nice, \& S. Thorsett (San Francisco: ASP), ASP Conf. Ser., 302, 145

Canizares, C. R., \& Winkler, P. F., Jr. 1981, ApJ, 246, L33

Chakrabarty, D., Pivovaroff, M. J., Hernquist, L. E., Jeremy, S. H., \& Narayan, R. 2001, ApJ, 548, 800

Cheng, K. S., \& Zhang, L. 1999, ApJ, 515, 337
Cheng, K. S., Ho, C. \& Ruderman, M. A. 1986, ApJ, 300, 500

Davis, J. E. 2001, ApJ, 562, 575

Davis, J. E. 2002, HEAD meeting in Albuquerque, http://space.mit.edu/CXC/analysis/davis/head2002/pileup.html De Jager, O. C., Swanepoel, J. W. H., \& Raubenheimer, B. C. 1989, A\&A, 221, 180

De Luca, A., Caraveo, P. A., Mereghetti, S., Negroni, M., \& Bignami, G. F. 2005, ApJ, 623, 1051

den Herder, et al. 2001, A\&A, 365, L7

Gaensler, B. M., Bock, D. C.-J., \& Stappers, B. W. 2000, ApJ, 537, L35

Gotthelf, E. V., Halpern, J. P., \& Seward, F. D. 2005, ApJ, in press

Helfand, D. J., \& Becker, R. H. 1984, Nature, 307, 215

Hui, C. Y., \& Cheng, K. S. 2004, ApJ, 608, 935

Iaria, R., Di Salvo, T., Burderi, L., \& Robba, N. R. 2001, ApJ, 548, 883

Jansen, F., et al. 2001, A\&A, 365, L1

Kaspi, V. M., Manchester, R. N., Johnston, S., Lyne, A. G., \& Amico, N. D' 1996, AJ, 111, 2028

Kaspi, V. M., Roberts, M. S. E., \& Harfing, A. 2004 [arXiv: astro-ph/0402136]

Kirsch, M. G. F., Becker, W., Benlloch-Garcia, S., et al. 2004, in X-Ray and Gamma-Ray Instrumentation for Astronomy XIII, ed. K. A. Flanagan, \& O. H. W. Siegmund, SPIE, 5165, 85

Kouveliotou, C., et al. 1998, Nature, 393, 235

Manchester, R. N., Hobbs, G. B., Teoh, A., \& Hobbs, M. 2005, AJ, in press (for recent update, please refer to http://www.atnf.csiro.au/research/pulsar/psrcat)

Manchester, R. N., et al. 2001, MNRAS, 328, 17

Mereghetti, S. 1998, Mem. Soc. Astron. Ital., 69, 819

Mewe, R., Gronenschild, E. H. B. M., \& van den Oord, G. H. J. 1985, A\&AS, 62, 197

Murray, S. S., et al. 1997, Proc. SPIE, 3114, 11

Murray, S. S., Ransom, S. M., Juda, M., Hwang, U., \& Holt, S. S. 2002, ApJ, 566,1039

Page, D. 1995, ApJ, 442, 273

Pavlov, G. G., Zavlin, V. E., \& Trümper, J. 1999, ApJ, 511, L45

Pavlov, G. G., Zavlin, V. E., Aschenbach, B., Trümper, J., \& Sanwal, D. 2000, ApJ, 531, L53

Pavlov, G. G., Sanwal, D., Garmire, G. P., \& Zavlin, V. E. 2002, in Neutron Stars in Supernova Remnants, ed. P. O. Slane, \& B. M. Gaensler, Astron. Soc. Pac. (San Francisco: ASP), ASP Conf. Ser., 271, 247

Petre, R., Canizares, C. R., Kriss, G. A., \& Winkler, P. F., Jr. 1982, ApJ, 258, 22 Petre, R., Becker, C. M., \& Winkler, P. F. 1996, ApJ, 465, L43

Pfeffermann, E., \& Aschenbach, B. 1996, in Roentgenstrahlung from the Universe, ed. H. H. Zimmermann, J. Trümper, \& H. Yorke (MPE Rep. 263, Garching: MPE), 267

Prakash, M., Ainsworth, T. L., \& Lattimer, J. M. 1988, Phys. Rev. Lett., 61, 2518 Reynoso, E. M., Dubner, G. M., Goss, W. M., \& Arnal, E. M. 1995, AJ, 110, 318 Reynoso, E. M., Green, A. J., Johnston, S., Dubner, G. M., Giacani, E. B., \& Goss, W. M. 2003, MNRAS, 345, 671

Seward, F. D., Slane, P. O., Smith, R. K., \& Sun, M. 2003, ApJ, 584, 414

Strüder, L., et al. 2001, A\&A, 365, L18

Swanepoel, J. W. H., de Beer, C. F., \& Loots, H. 1996, ApJ, 467, 261

Tananbaum, H. 1999, IAU Circ., 7246

Tennant, A. F., Becker, W., Juda, M., et al. 2001, ApJ, 554, L173

Thompson, C. 2000, in Pulsar Astronomy-2000 and Beyond, ed. M. Kramer, N. Wex, \& N. Wielebinski (San Francisco: ASP), IAU Colloq., 177, ASP Conf. Ser. 202, 669

Tuohy, I. R., \& Garmire, G. P. 1980, ApJ, 239, L107

Turner, M. J. L., et al. 2001, A\&A, 365, L27

van Paradijs, J., Taam, R. E., \& van den Heuvel, E. P. J. 1995, A\&A, 299, L41

Weisskopf, M. C. 2004, Proc. SPIE, 5488, 25

Winkler, P. F., \& Kirshner, R. P. 1985, ApJ, 299, 981

Winkler, P. F., Canizares, C. R., Clark, G. W., et al. 1981, ApJ, 246, L27

Winkler, P. F., Tuttle, J. H., Kirshner, R. P., \& Irwin, M. J. 1988, in Supernova Remnants and the Interstellar Medium, ed. R. S. Roger, \& T. Landecker (Cambridge: Cambridge Univ. Press), 65

Winkler, P. F., Kirshner, R. P., Hughes, J. P., \& Heathcote, S. R. 1989, Nature, 337,48

Woods, P. M., et al. 2002, ApJ, 576, 381

Yakolev, D. G., Gnedin, O. Y., Kaminker, A. D., Levenfish, K. P., \& Potekhin, A. Y. 2004, AdSpR, 33, 523

Zavlin, V. E., Trümper, J., \& Pavlov, G. G. 1999, ApJ, 525, 959

Zavlin, V. E., Pavlov, G. G., \& Sanwal, D. 2004, ApJ, 606, 444

Zombeck, M. V., Chappell, J. H., Kenter, A. T., et al. 1995, Proc. SPIE, 2518, 96 